\title{
A Quantum Cosmological Model With Static and Dynamic Wormholes
}

\author{
N.A. Lemost and G. A. Monerat \\ Departamento de Física, Universidade Federal Fluminense \\ CEP 24210-340, Niterói, Rio de Janeiro, Brazil
}

\begin{abstract}
Quantization is performed of a Friedmann-Robertson-Walker universe filled with a conformally invariant scalar field and a perfect fluid with equation of state $p=\alpha \rho$. A well-known discrete set of static quantum wormholes is shown to exist for radiation $(\alpha=1 / 3)$, and a novel continuous set is found for cosmic strings $(\alpha=-1 / 3)$, the latter states having throat radii of any size. In both cases wave-packet solutions to the Wheeler-DeWitt equation are obtained with all the properties of evolving quantum wormholes. In the case of a radiation fluid, a detailed analysis of the quantum dynamics is made in the context of the Bohm-de Broglie interpretation. It is shown that a repulsive quantum force inversely proportional to the cube of the scale factor prevents singularities in the quantum domain. For the states considered, there are no particle horizons either.
\end{abstract}

PACS number(s): 04.20.Cv., 04.20.Me

KEY WORDS: Quantum cosmology; Bohm-de Broglie interpretation; wormholes.

\section{Introduction}

Quantum cosmology and speculations about quantum effects in the very early Universe began with the work of DeWitt [1]. Since then, many investigators have been facing the difficult task of giving a reasonable description of the Planck era. Friedmann-RobertsonWalker (FRW) models have been intensely studied, and it has been found that some of them possess wormhole quantum states. Quantum wormholes are solutions to the Wheeler-DeWitt equation which remain regular as the three-geometry collapses to zero and are exponentially damped for large three-geometries [2]. In quantum cosmology wormhole states are not restricted to exotic types of matter fields, and in fact they exist in models with a simple matter content, such as dust and a conformally coupled scalar field [3].

Recently, in collaboration with J. C. Fabris and F. G. Alvarenga, we quantized a FRW model having as matter content a perfect fluid with an arbitrary barotropic equation of state 《4. Wave packets were constructed and the behaviour of the scale factor discussed

\footnotetext{
${ }^{1}$ e-mail: nivaldo@if.uff.br

${ }^{2}$ e-mail: monerat@if.uff.br
} 
according to the many-worlds and the Bohm-de Broglie interpretations. Since fundamental fields are believed to play an essential role in the dynamics of the early Universe, in the present paper we study the consequences of adding a conformal scalar field to the matter content. The treatment relies on Schutz's canonical formalism [5], which describes a relativistic fluid interacting with the gravitational field and endows the fluid with dynamical degrees of freedom. The quantum properties of the model are investigated on the basis of the associated Wheeler-DeWitt equation. The super-Hamiltonian constraint turns out to be linear in one of the momenta, and a time variable can be naturally introduced, reducing the Wheeler-DeWitt equation to a bona fide Schrödinger equation. The presence of the conformal scalar field gives rise to significant changes in the quantum dynamics. A well-known discrete set of static wormhole wave functions exists in the case of radiation. In the case of cosmic strings a new continuous family of quantum wormhole states is found whose throat radii can be arbitrarily small or arbitrarily large. Both for cosmic strings and radiation, wave packets are obtained with the properties of evolving quantum wormholes. For a radiation fluid we manage to construct the exact propagator, which allows us to study the evolution of initial states of the Gaussian type. We show that a strong repulsive quantum force, which is inversely proportional to the cube of the scale factor, prevents the appearence of singularities. There is no particle horizon either.

This paper is organized as follows. In Section 2 a Hamiltonian treatment is given to a FRW universe filled with a perfect fluid (with an arbitrary barotropic equation of state $p=\alpha \rho$ ) and a conformally invariant scalar field. In Section 3 the classical equations of motion are solved for some particular values of $\alpha$. In Section 4 the WheelerDeWitt equation is constructed. Static quantum wormholes are obtained in Section 5 for radiation and cosmic strings. In Section 6 dynamic wormholes are obtained for cosmic strings and radiation, in the latter case by means of the exact propagator to the WheelerDeWitt equation. The rest of the paper deals only with radiation. The Bohm-de Broglie interpretation is employed in Section 7 to study the quantum dynamics, and the role of the quantum force to prevent singularities is highlighted. Section 8 is dedicated to final comments.

\section{FRW Model With Perfect Fluid and Conformal Scalar Field}

A homogeneous and isotropic cosmological model is characterized by the FriedmannRobertson-Walker metric

$$
d s^{2}=-N(t)^{2} a(t)^{2} d t^{2}+a(t)^{2} \sigma_{i j} d x^{i} d x^{j},
$$

where $\sigma_{i j}$ denotes the metric for a 3 -space of constant curvature $k=+1,0$ or -1 , and the lapse function has been conveniently parametrized as $N(t) a(t)$.

In units such that $c=16 \pi G=1$, the pure gravitational action is

$$
S_{g}=\int_{M} d^{4} x \sqrt{-g} R+2 \int_{\partial M} d^{3} x \sqrt{h} K
$$


where $K$ is the trace of the extrinsic curvature $K_{i j}$ of the boundary $\partial M$ of the space-time manifold $M$. The matter content is a perfect fluid plus a scalar field conformally coupled to gravity. The action associated with the sources of gravity is

$$
S_{m}=\int_{M} d^{4} x \sqrt{-g} p-\frac{1}{2} \int_{M} d^{4} x \sqrt{-g}\left(\partial_{\mu} \phi \partial^{\mu} \phi+\frac{1}{6} R \phi^{2}\right)-\frac{1}{12} \int_{\partial M} d^{3} x \sqrt{h} K \phi^{2} .
$$

Schutz's canonical formalism [0] makes use of a representation for the four-velocity of the fluid as $U_{\nu}=\frac{1}{\mu}\left(\epsilon,_{\nu}+\zeta \beta,_{\nu}+\theta S,_{\nu}\right)$, where $\epsilon, \zeta, \beta, \theta, S$ are five velocity potentials. The specific enthalpy $\mu$ is expressed in terms of the velocity potentials by means of the normalization condition $U^{\nu} U_{\nu}=-1$. The potential $S$ is the specific entropy, and in FRW models the potentials $\zeta$ and $\beta$ are zero.

Compatibility with the homogeneous spacetime metric is guaranteed by taking the scalar field and the velocity potentials as functions of $t$ only. Taking $p=\alpha \rho$ as equation of state for the fluid, and performing an ADM reduction described in detail in [6, 7], we can write the total action $S_{T}=S_{g}+S_{m}$ as [6, 0, 8]

$$
S_{T}=\int d t\left(p_{a} \dot{a}+p_{\Phi} \dot{\Phi}+p_{\epsilon} \dot{\epsilon}+p_{S} \dot{S}-N \mathcal{H}\right)
$$

where we have used the constraint $p_{\theta}=0$ and redefined the scalar field by means of $\Phi=a \phi$. The super-Hamiltonian $\mathcal{H}$ is given by

$$
\mathcal{H}=-\frac{p_{a}^{2}}{24}-6 k a^{2}+p_{\epsilon}^{\alpha+1} a^{1-3 \alpha} e^{S}+\frac{p_{\Phi}^{2}}{24}+6 k \Phi^{2} .
$$

The canonical transformation [4]

$$
T=p_{S} e^{-S} p_{\epsilon}^{-(\alpha+1)}, \quad p_{T}=e^{S} p_{\epsilon}^{\alpha+1}, \quad \bar{\epsilon}=\epsilon-(\alpha+1) \frac{p_{S}}{p_{\epsilon}}, \quad p_{\bar{\epsilon}}=p_{\epsilon}
$$

reduces the super-Hamiltonian to

$$
\mathcal{H}=-\frac{p_{a}^{2}}{24}+\frac{p_{\Phi}^{2}}{24}-6 k a^{2}+6 k \Phi^{2}+a^{1-3 \alpha} p_{T} .
$$

Since $\bar{\epsilon}$ and $p_{\bar{\epsilon}}$ do not appear in the super-Hamiltonian (7), they do not represent a dynamical degree of freedom and may be simply dropped from the action, which takes the reduced form

$$
S=\int d t\left(p_{a} \dot{a}+p_{\Phi} \dot{\Phi}+p_{T} \dot{T}-N \mathcal{H}\right)
$$

\section{The Classical Equations of Motion}

The variational principle $\delta S=0$ leads to the classical equations of motion

$$
\begin{gathered}
\dot{a}=-\frac{N}{12} p_{a} \quad, \quad \dot{\Phi}=\frac{N}{12} p_{\Phi} \quad, \quad \dot{T}=N a^{1-3 \alpha}, \\
\dot{p}_{a}=N\left[12 k a-(1-3 \alpha) a^{-3 \alpha} p_{T}\right] \quad, \quad \dot{p}_{\Phi}=-12 N k \Phi \quad, \quad \dot{p}_{T}=0
\end{gathered}
$$


supplemented by the super-Hamiltonian constraint

$$
-\frac{p_{a}^{2}}{24}+\frac{p_{\Phi}^{2}}{24}-6 k a^{2}+6 k \Phi^{2}+a^{1-3 \alpha} p_{T}=0
$$

It follows that $p_{T}=$ constant, and in the conformal-time gauge $(N=1)$ we have

$$
\ddot{\Phi}+k \Phi=0 \quad, \quad \dot{T}=a^{1-3 \alpha} \quad, \quad \ddot{a}=-k a+\frac{1-3 \alpha}{12} a^{-3 \alpha} p_{T} .
$$

The cases $\alpha=-1$ and $\alpha=0$ have been previously studied in [3]. If $\alpha=1 / 3$, the equation of motion for the scale factor becomes identical to that satisfied by $\Phi$. If $\alpha \neq 1 / 3$, equation (11) for the scale factor corresponds to Newton's equation of motion for a particle of unit mass under a force whose potential is $V(a)=k a^{2} / 2+\ell a^{1-3 \alpha}$, where $\ell=-p_{T} / 12$ is a constant. Making use of "energy" conservation we find

$$
t=\int \frac{d a}{\sqrt{\mathcal{E}-k a^{2}-\ell a^{1-3 \alpha}}},
$$

where $\mathcal{E}$ is an integration constant. The above integral can be expressed in terms of elementary functions in the cases of radiation $(\alpha=1 / 3)$, stiff matter $(\alpha=1)$ and cosmic strings $(\alpha=-1 / 3)$. In other physically interesting cases such as dust $(\alpha=0)$, domain walls $(\alpha=-2 / 3)$ and vacuum $(\alpha=-1)$ the integral is given by Jacobi elliptic functions.

For future reference we solve the classical equations of motion in the radiation case ( $\alpha=1 / 3)$. In the conformal-time gauge $(N=1)$ eqs.(9) are solved by (the three lines below correspond to $k=-1,0,1$, respectively)

$$
\begin{aligned}
& a(\tau)=c_{1} \sinh \tau+c_{2} \cosh \tau, \quad \Phi(\tau)=c_{3} \sinh \tau+c_{4} \cosh \tau, \\
& a(\tau)=c_{1} \tau+c_{2}, \Phi(\tau)=c_{3} \tau+c_{4}, \\
& a(\tau)=c_{1} \sin \tau+c_{2} \cos \tau, \quad \Phi(\tau)=c_{3} \sin \tau+c_{4} \cos \tau,
\end{aligned}
$$

where $c_{1}, c_{2}, c_{3}, c_{4}$ and $p_{T}$ are integration constants. For $k=1$ both $a(\tau)$ and $\Phi(\tau)$ are oscillating functions. The cases $k=-1$ and $k=0$ correspond to an ever-expanding Universe.

\section{Quantization}

The Wheeler-DeWitt quantization scheme consists in promoting the canonical momenta to operators according to

$$
p_{a} \rightarrow-i \frac{\partial}{\partial a}, p_{\Phi} \rightarrow-i \frac{\partial}{\partial \Phi}, p_{T} \rightarrow-i \frac{\partial}{\partial T}
$$

and, with the corresponding super-Hamiltonian operator $\hat{\mathcal{H}}$, forming the Wheeler-DeWitt equation

$$
\hat{\mathcal{H}} \Psi(a, \Phi, T)=0
$$


where $\Psi(a, \Phi, T)$ is called the wave function of the Universe. In our case, the WheelerDeWitt equation associated with eq.(10) takes the form of the Schrödinger-like equation

$$
\left(\frac{1}{24} \frac{\partial^{2}}{\partial a^{2}}-6 k a^{2}\right) \Psi(a, \Phi, T)-\left(\frac{1}{24} \frac{\partial^{2}}{\partial \Phi^{2}}-6 k \Phi^{2}\right) \Psi(a, \Phi, T)=i a^{1-3 \alpha} \frac{\partial}{\partial T} \Psi(a, \Phi, T) .
$$

For the sake of convenience, let us perform the reparametrization

$$
a=\frac{R}{\sqrt{12}}, \Phi=\frac{\chi}{\sqrt{12}}, \tau=-T,
$$

which casts equation (16) into the form

$$
\left[\left(-\frac{1}{2} \frac{\partial^{2}}{\partial R^{2}}+\frac{k}{2} R^{2}\right)-\left(-\frac{1}{2} \frac{\partial^{2}}{\partial \chi^{2}}+\frac{k}{2} \chi^{2}\right)\right] \Psi(R, \chi, \tau)=i\left(\frac{R}{\sqrt{12}}\right)^{1-3 \alpha} \frac{\partial \Psi(R, \chi, \tau)}{\partial \tau} .
$$

Eq.(18) can be written as $i \partial \Psi / \partial t=\hat{H} \Psi$ with a self-adjoint Hamiltonian operator if the inner product is 4 ]

$$
(\Psi, \Phi)=\int_{-\infty}^{\infty} d \chi \int_{0}^{\infty} d R R^{1-3 \alpha} \Psi(R, \chi)^{*} \Phi(R, \chi)
$$

\section{$5 \quad$ Static Quantum Wormholes}

For the radiation case $(\alpha=1 / 3)$ and $k=1$ the stationary solutions of the WheelerDeWitt equation (18) take the form $\Psi(R, \chi, \tau)=\varphi_{n}(R) \varphi_{m}(\chi) e^{-i E \tau}$ where $\varphi_{n}$ is the n-th eigenfunction of the harmonic oscillator and $E=E_{n m}=n-m$, with $n, m=0,1,2, \ldots$ The wave functions $\psi_{n m}(R, \chi)=\varphi_{n}(R) \varphi_{m}(\chi)$ form a well-known set of wormhole quantum states [2, 9, 10, 11].

We now show that static wormholes also exist for cosmic strings and $k=1$. With $\alpha=-1 / 3$ the Wheeler-DeWitt equation (18) becomes

$$
\left(-\frac{1}{2} \frac{\partial^{2}}{\partial R^{2}}+\frac{1}{2} k R^{2}\right) \Psi(R, \chi, \tau)-\left(-\frac{1}{2} \frac{\partial^{2}}{\partial \chi^{2}}+\frac{1}{2} k \chi^{2}\right) \Psi(R, \chi, \tau)=i \frac{R^{2}}{12} \frac{\partial}{\partial \tau} \Psi(R, \chi, \tau) .
$$

Writing $\Psi=e^{-i E \tau} \psi$ we find

$$
\frac{1}{2}\left[-\frac{\partial^{2} \psi}{\partial R^{2}}+k R^{2} \psi\right]-\frac{1}{2}\left[-\frac{\partial^{2} \psi}{\partial \chi^{2}}+k \chi^{2} \psi\right]=E \frac{R^{2}}{12} \psi
$$

Applying the method of separation of variables with $\psi(R, \chi)=X(R) Y(\chi)$ we get

$$
\frac{1}{2 X}\left[-X^{\prime \prime}+k R^{2} X\right]-E \frac{R^{2}}{12}=\frac{1}{2 Y}\left[-Y^{\prime \prime}+k \chi^{2} Y\right]=\frac{A}{2},
$$

where $A$ is a separation constant. Thus we have the following ordinary differential equations: 


$$
\begin{gathered}
X^{\prime \prime}+\left(A-k R^{2}+E \frac{R^{2}}{6}\right) X=0, \\
Y^{\prime \prime}+\left(A-k \chi^{2}\right) Y=0 .
\end{gathered}
$$

With $k=1$ and $A=0$ these equations reduce to

$$
\begin{gathered}
X^{\prime \prime}-\left(1-\frac{E}{6}\right) R^{2} X=0, \\
Y^{\prime \prime}-\chi^{2} Y=0 .
\end{gathered}
$$

The general solution to these equations which is regular everywhere and exponentially damped for both large scale factor and large scalar field is [12]

$$
\begin{gathered}
X(R)=R^{1 / 2} K_{1 / 4}\left(\sqrt{1-E / 6} R^{2} / 2\right), \\
Y(\chi)=|\chi|^{1 / 2} K_{1 / 4}\left(\chi^{2} / 2\right),
\end{gathered}
$$

where $E<6$ and $K_{\nu}$ is the modified Bessel function of the second kind, of order $\nu$.

Thus, the static wormhole wave functions in this case are

$$
\psi_{E}(R, \chi)=N_{E}(|\chi| R)^{1 / 2} K_{1 / 4}\left(\chi^{2} / 2\right) K_{1 / 4}\left(\sqrt{1-E / 6} R^{2} / 2\right),
$$

with $E<6$. These wave functions are normalizable in the inner product (19). The normalization constant is found to be [Ref. [13], formulas 6.521(3) and 6.576(4)]

$$
N_{E}=\frac{2}{\pi^{3 / 2}} \sqrt{1-E / 6} .
$$

Thus, in the case of a fluid of cosmic strings there exists a continuous family of wormhole quantum states. Because the Hilbert space of states is separable, the wave functions (29) cannot be mutually ortogonal, just like the elements of the overcomplete set of coherent states of the harmonic oscillator. Indeed, we have

$$
\left(\psi_{E^{\prime}}, \psi_{E}\right)=\frac{4\left(1-E^{\prime} / 6\right)^{3 / 8}(1-E / 6)^{3 / 8}}{\left[\left(1-E^{\prime} / 6\right)^{1 / 4}+(1-E / 6)^{1 / 4}\right]\left[\left(1-E^{\prime} / 6\right)^{1 / 2}+(1-E / 6)^{1 / 2}\right]} .
$$

The expectation value of the scale factor in the state (29) is

$$
\langle R\rangle=\frac{2^{7 / 2}}{3 \pi} \frac{\Gamma(1 / 4)^{2}}{(1-E / 6)^{1 / 4}},
$$

which may be interpreted as the radius of the throat. Since $-\infty<E<6$, static wormhole universes with arbitrarily small or arbitrarily large throat radii are allowed. From equations (14), (17) and $\Psi=e^{-i E \tau} \psi$ it follows that $p_{T}=E$. For $E<6$ the classical equations of motion (11) with $\alpha=-1 / 3$ have no static solution for the scale factor. Thus, the quantum regime in the state (29) is such that the classical evolution of the universe is halted. 


\section{Evolving Quantum Wormholes}

In the case of cosmic strings, let us consider $E>6$. Setting $E=6\left(1+\epsilon^{2}\right)$ with $\epsilon>0$, a solution to eq. (25) is

$$
X(R)=R^{1 / 2} J_{1 / 4}\left(\epsilon R^{2} / 2\right),
$$

where $J_{\nu}$ is the Bessel function of the first kind, of order $\nu$. The stationary wave functions

$$
\psi_{\epsilon}(R, \chi)=(|\chi| R)^{1 / 2} K_{1 / 4}\left(\chi^{2} / 2\right) J_{1 / 4}\left(\epsilon R^{2} / 2\right)
$$

do not represent physical states because they are not normalizable in the inner product (19). Normalizable wave packets may be constructed by writing

$$
\begin{gathered}
\Psi(R, \chi, \tau)=\int_{0}^{\infty} C(\epsilon) e^{-i E \tau} \psi_{\epsilon}(R, \chi) d \epsilon \\
=e^{-6 i \tau}(|\chi| R)^{1 / 2} K_{1 / 4}\left(\chi^{2} / 2\right) \int_{0}^{\infty} C(\epsilon) e^{-6 i \tau \epsilon^{2}} J_{1 / 4}\left(\epsilon R^{2} / 2\right) d \epsilon .
\end{gathered}
$$

The choice

$$
C(\epsilon)=\epsilon^{5 / 4} e^{-\gamma \epsilon^{2}}
$$

with $\gamma$ a positive constant, leads to [Ref.[13], formula 6.631(4)]

$$
\Psi(R, \chi, \tau)=\frac{e^{-6 i \tau}}{(\gamma+6 i \tau)^{5 / 4}}|\chi|^{1 / 2} K_{1 / 4}\left(\chi^{2} / 2\right) R \exp \left[-\frac{R^{4}}{16(\gamma+6 i \tau)}\right]
$$

up to a normalization factor. The expectation value of the scale factor in the state (37) is

$$
\langle R\rangle(\tau)=\frac{2^{7 / 4} \sqrt{\pi}}{\Gamma(1 / 4)}\left(\frac{\gamma^{2}+36 \tau^{2}}{\gamma}\right)^{1 / 4}
$$

Note that the mean radius of the Universe grows without bound in spite of the fact that the spatial geometry of the Universe is closed $(k=1)$. Taking $\gamma$ sufficiently small, so that the wave packet is very well localized near $R=0$ at $\tau=0$, the expectation value of the scale factor becomes as large as one pleases immediately after $\tau=0$. In other words, it is nearly certain that the Universe will have an enormous radius at any positive time. This was called "inflation without inflation" in [14, 15].

Let us take up again the radiation case. With $\alpha=1 / 3$ we can put equation (18) in the form

$$
i \frac{\partial}{\partial \tau} \Psi(R, \chi, \tau)=\hat{H} \Psi(R, \chi, \tau) \equiv\left(\hat{H}_{R}-\hat{H}_{\chi}\right) \Psi(R, \chi, \tau)
$$

where

$$
\hat{H}_{R}=-\frac{1}{2} \frac{\partial^{2}}{\partial R^{2}}+\frac{k}{2} R^{2} \quad, \quad \hat{H}_{\chi}=-\frac{1}{2} \frac{\partial^{2}}{\partial \chi^{2}}+\frac{k}{2} \chi^{2} .
$$

The Hamiltonian operator $\hat{H}$ is self-adjoint in the inner product

$$
(\Psi, \Phi)=\int_{-\infty}^{\infty} d \chi \int_{0}^{\infty} d R \Psi(R, \chi)^{*} \Phi(R, \chi)
$$


as long as its domain is restricted to those wave functions such that $\Psi(0, \chi)=0$ or $\psi^{\prime}(0, \chi)=0$, where the prime means partial derivative with respect to $R$ [15]. Except for this domain restriction, $\hat{H}$ is the difference between: Hamiltonians of two harmonic oscillators $(k=1)$; Hamiltonians of two free particles $(k=0)$; or Hamiltonians of two inverted oscillators $(k=-1)$.

The propagator for (39) is

$$
G\left(\chi, R ; \chi^{\prime}, R^{\prime} ; \tau\right)=G_{R}\left(R ; R^{\prime} ; \tau\right) G^{*}\left(\chi ; \chi^{\prime} ; \tau\right)
$$

where $G$ is the usual harmonic-oscillator propagator. As concerns $G_{R}$, some care must be exercised since $R$ takes values only on the half-line $R \geq 0$. For the sake of simplicity, we consider only those wave functions that satisfy the condition $\Psi^{\prime}(0, \chi, \tau)=0$. For this boundary condition, the propagator is given by [15]

$$
G_{R}\left(R, R^{\prime} ; \tau\right)=G\left(R ; R^{\prime} ; \tau\right)+G\left(R ;-R^{\prime} ; \tau\right)
$$

where

$$
G\left(x, x^{\prime} ; \tau\right)=\left(\frac{\sqrt{k}}{2 \pi i \sin \sqrt{k} \tau}\right)^{1 / 2} \exp \left\{\frac{i \sqrt{k}}{2 \sin \sqrt{k} \tau}\left[\left(x^{2}+x^{\prime 2}\right) \cos \sqrt{k} \tau-2 x x^{\prime}\right]\right\}
$$

is the propagator for a one-dimensional harmonic oscillator with mass $m=1$ and frequency $w=\sqrt{k}$.

The quantum dynamics of the models will be studied by following the time evolution of the initial Gaussian wave function

$$
\Psi(\chi, R, 0)=\sqrt{\frac{4 \sqrt{\sigma \beta}}{\pi}} e^{-\sigma R^{2}-\beta \chi^{2}},
$$

where $\sigma$ and $\beta$ are positive constants. Making use of

$$
\Psi(\chi, R, \tau)=\int_{-\infty}^{\infty} d \chi^{\prime} \int_{0}^{\infty} d R^{\prime} G\left(\chi, R ; \chi^{\prime}, R^{\prime} ; \tau\right) \Psi\left(\chi^{\prime}, R^{\prime}, 0\right)
$$

the wave packet takes the form

$$
\begin{aligned}
\Psi(R, \chi, \tau) & =\sqrt{\frac{4 \sqrt{\sigma \beta}}{\pi}} \sqrt{\frac{k}{\cos ^{2}(\sqrt{k} \tau)[2 \sigma \tan (\sqrt{k} \tau)-i \sqrt{k}][2 \beta \tan (\sqrt{k} \tau)+i \sqrt{k}]}} \\
& \times \exp \left\{-\frac{i \sqrt{k}}{2 \tan (\sqrt{k} \tau)}\left[1-\frac{i \sqrt{k}}{\cos ^{2}(\sqrt{k} \tau)[2 \beta \tan (\sqrt{k} \tau)+i \sqrt{k}]}\right] \chi^{2}\right. \\
& \left.+\frac{i \sqrt{k}}{2 \tan (\sqrt{k} \tau)}\left[1+\frac{i \sqrt{k}}{\cos ^{2}(\sqrt{k} \tau)[2 \sigma \tan (\sqrt{k} \tau)-i \sqrt{k}]}\right] R^{2}\right\} .
\end{aligned}
$$




\section{The Bohm-de Broglie Interpretation}

The Bohm-De Broglie interpretation [16] is an alternative interpretation of quantum mechanics which allows the treatment of a unique system such as the Universe, which cannot be repeatedly prepared in the same state as required by the standard statistical interpretation. The Bohm-De Broglie interpretation starts by writing the wave function of the Universe in the form

$$
\Psi=\Theta \exp (i \mathcal{S})
$$

where $\Theta$ and $\mathcal{S}$ are real functions. Then, inserting (48) into the Wheeler-DeWitt equation (16) with $\alpha=1 / 3$, there results

$$
\begin{aligned}
& \frac{\partial \mathcal{S}}{\partial \tau}+\frac{1}{2}\left[\left(\frac{\partial \mathcal{S}}{\partial \chi}\right)^{2}-\left(\frac{\partial \mathcal{S}}{\partial R}\right)^{2}\right]+V+Q=0 \\
& \frac{\partial \Theta}{\partial \tau}+\left[\left(\frac{\partial \Theta}{\partial \chi}\right)\left(\frac{\partial \mathcal{S}}{\partial \chi}\right)-\left(\frac{\partial \Theta}{\partial R}\right)\left(\frac{\partial \mathcal{S}}{\partial R}\right)\right]+\frac{\Theta}{2}\left[\frac{\partial^{2} \mathcal{S}}{\partial \chi^{2}}-\frac{\partial^{2} \mathcal{S}}{\partial R^{2}}\right]=0,
\end{aligned}
$$

where $V$ and $Q$ are the classical and quantum potentials, respectively:

$$
V=\frac{1}{2} k\left(R^{2}-\chi^{2}\right) \quad, \quad Q=-\frac{1}{2 \Theta}\left[\frac{\partial^{2} \Theta}{\partial R^{2}}-\frac{\partial^{2} \Theta}{\partial \chi^{2}}\right] .
$$

Let us first examine the case of vanishing spatial curvature $(k=0)$.

\subsection{The Flat Case}

The wave packet is obtained by taking the limit as $k \rightarrow 0$ of the wave function (47), and it takes the form

$$
\Psi(R, \chi, \tau)=\sqrt{4 \frac{\sqrt{\sigma \beta}}{\pi}} \Psi_{1}(R, \tau) \Psi_{2}(\chi, \tau)
$$

where

$$
\begin{aligned}
& \Psi_{1}(R, \tau)=\sqrt{\frac{1}{2 \sigma \tau-i}} \exp \left\{\left(\frac{i}{2 \tau} \frac{2 \sigma \tau}{2 \sigma \tau-i}\right) R^{2}\right\}, \\
& \Psi_{2}(\chi, \tau)=\sqrt{\frac{1}{2 \beta \tau+i}} \exp \left\{\left(-\frac{i}{2 \tau} \frac{2 \beta \tau}{2 \beta \tau+i}\right) \chi^{2}\right\} .
\end{aligned}
$$

The wave function (51) may be written in the form (48) with the functions $\Theta$ and $\mathcal{S}$ given by

$$
\begin{aligned}
& \mathcal{S}=\frac{2 \sigma^{2} \tau}{1+4 \sigma^{2} \tau^{2}} R^{2}-\frac{2 \beta^{2} \tau}{1+4 \beta^{2} \tau^{2}} \chi^{2}+f_{0}(\tau), \\
& \Theta=g_{0}(\tau) \exp \left\{-\frac{\sigma R^{2}}{1+4 \sigma^{2} \tau^{2}}-\frac{\beta \chi^{2}}{1+4 \beta^{2} \tau^{2}}\right\},
\end{aligned}
$$

where $f_{0}$ and $g_{0}$ are functions of $\tau$ alone that play no role in the subsequent discussion. From this we can find the Bohmian trajectories for the scale factor $a(\tau)$ and the scalar 
field $\Phi(\tau)$, recalling that $a, \Phi, R$ and $\chi$ are related by (17), and also that in the $\tau$-gauge one must take $N=-1$ in Hamilton's equations (9). The Bohmian trajectories are the solutions of the differential equations

$$
p_{R}=\frac{\partial \mathcal{S}}{\partial R}, \quad p_{\chi}=\frac{\partial \mathcal{S}}{\partial \chi}
$$

Taking into account the previous remark on the lapse function, the classical equations of motion and the relations (17), we get

$$
\dot{R}(\tau)=\frac{4 \sigma^{2} \tau}{1+4 \sigma^{2} \tau^{2}} R(\tau)
$$

An immediate integration yields

$$
R(\tau)=A_{0} \sqrt{1+4 \sigma^{2} \tau^{2}}
$$

where $A_{0}$ is a positive constant of integration. There is no singularity since the Bohmian trajectories $R(\tau)$ never reach $R=0$.

In a similar fashion, we find for the scalar field

$$
\dot{\chi}(\tau)=\frac{4 \beta^{2} \tau}{1+4 \beta^{2} \tau^{2}} \chi(\tau)
$$

whose solution is

$$
\chi(\tau)=B_{0} \sqrt{1+4 \beta^{2} \tau^{2}},
$$

where $B_{0}$ is a real constant of integration. The probability distribution of $\chi$ derived from (54) is an even function of $\chi$. Thus, averaging over $B_{0}$ we obtain the expectation value $\langle\chi\rangle=0$, as it should.

The quantum potential $Q$ is found by inserting (54) into (50):

$$
Q(R, \chi, \tau)=\frac{\sigma}{1+4 \sigma^{2} \tau^{2}}-\frac{\beta}{1+4 \beta^{2} \tau^{2}}-\frac{2 \sigma^{2} R^{2}}{\left(1+4 \sigma^{2} \tau^{2}\right)^{2}}+\frac{2 \beta^{2} \chi^{2}}{\left(1+4 \beta^{2} \tau^{2}\right)^{2}}
$$

The quantum force associated with the time evolution of the scale factor is

$$
F_{R}^{(Q)}=-\frac{\partial Q}{\partial R}=\frac{4 \sigma^{2} R}{\left(1+4 \sigma^{2} \tau^{2}\right)^{2}} .
$$

With the help of the trajectory (57), the quantum force $F_{R}$ can be expressed as a function of $R$ only:

$$
F_{R}^{(Q)}(R)=\frac{C}{R^{3}}
$$

where $C$ is a positive constant. This force is always repulsive away from $R=0$, is strongest when $R(\tau)$ is minimum and is responsible for the avoidance of the singularity in the quantum domain. 


\subsection{The Case of Positive Spatial Curvature}

Now the wave function (47) takes the form

$$
\Psi(R, \chi, \tau)=\sqrt{\frac{4 \sqrt{\sigma \beta}}{\pi}} \Theta \exp (i \mathcal{S})
$$

with $\mathcal{S}$ and $\Theta$ given by

$$
\Theta=g_{1}(\tau) \exp \left\{-\frac{\sigma R^{2}}{\cos ^{2} \tau+4 \sigma^{2} \sin ^{2} \tau}-\frac{\beta \chi^{2}}{\cos ^{2} \tau+4 \beta^{2} \sin ^{2} \tau}\right\}
$$

and

$$
\mathcal{S}=f_{1}(\tau)+\frac{R^{2}}{2 \tan \tau}\left[1-\frac{1}{\cos ^{2} \tau+4 \sigma^{2} \sin ^{2} \tau}\right]-\frac{\chi^{2}}{2 \tan \tau}\left[1-\frac{1}{\cos ^{2} \tau+4 \beta^{2} \sin ^{2} \tau}\right] .
$$

The Bohmian trajectories are determined by

$$
\begin{aligned}
& \dot{R}(\tau)=\frac{1}{\tan \tau}\left[1-\frac{1}{\cos ^{2} \tau+4 \sigma^{2} \sin ^{2} \tau}\right] R(\tau), \\
& \dot{\chi}(\tau)=\frac{1}{\tan \tau}\left[1-\frac{1}{\cos ^{2} \tau+4 \beta^{2} \sin ^{2} \tau}\right] \chi(\tau) .
\end{aligned}
$$

Integration yields

$$
R(\tau)=A_{+} \sqrt{\cos ^{2} \tau+4 \sigma^{2} \sin ^{2} \tau}, \chi(\tau)=B_{+} \sqrt{\cos ^{2} \tau+4 \beta^{2} \sin ^{2} \tau}
$$

where $A_{+}$and $B_{+}$are constants of integration, with $A_{+}>0$. Both $R$ and $\chi$ oscillate eternally between minimum and maximum values.

The quantum potential is

$$
Q(R, \chi, \tau)=h(\tau)-\frac{2 \sigma^{2} R^{2}}{\left(\cos ^{2} \tau+4 \sigma^{2} \sin ^{2} \tau\right)^{2}}+\frac{2 \beta^{2} \chi^{2}}{\left(\cos ^{2} \tau+4 \beta^{2} \sin ^{2} \tau\right)^{2}},
$$

from which it is easily seen that the quantum force is again of the form (62).

Only the interval $[0, \pi]$ is physically acceptable for $\tau$ at the classical level, corresponding to the initial and final singularities. In the quantum realm, however, $\tau$ may take values over the whole real line, there being no singularity in virtue of the repulsive character of the quantum force. Differently from the flat case, in which only the quantum potential exists, in the present case $(k=1)$ the classical an quantum potentials compete. However, as equation (62) shows, the repulsive quantum force is much stronger that the classical force near the singularity. 


\subsection{The Case of Negative Spatial Curvature}

The results for $k=-1$ follow from those for $k=1$ by simply replacing the trigonometric functions by the corresponding hyperbolic functions. The Bohmian trajectories satisfy

$$
\begin{aligned}
& \dot{R}(\tau)=\frac{1}{\tanh \tau}\left[1-\frac{1}{\cosh ^{2} \tau+4 \sigma^{2} \sinh ^{2} \tau}\right] R(\tau), \\
& \dot{\chi}(\tau)=\frac{1}{\tanh \tau}\left[1-\frac{1}{\cosh ^{2} \tau+4 \beta^{2} \sinh ^{2} \tau}\right] \chi(\tau),
\end{aligned}
$$

which are solved by

$$
R(\tau)=A_{-} \sqrt{\cosh ^{2} \tau+4 \sigma^{2} \sinh ^{2} \tau}, \chi(\tau)=B_{-} \sqrt{\cosh ^{2} \tau+4 \beta^{2} \sinh ^{2} \tau} .
$$

with $A_{-}>0$. Once again the quantum force as a function of $R$ is given by (62).

\section{Conclusion and Final Remarks}

In this work we quantized Friedmann-Robertson-Walker cosmological models having for matter content a perfect fluid and a conformal scalar field. We found static wormhole wave functions in the cases of radiation and cosmic strings. In the radiation case the discrete set of wormhole quantum states was previously known in the literature. In the case of cosmic strings we found a new continuous family of static quantum wormholes with arbitrary throat radii. The members of this continuous set of finite-norm wave functions are not orthogonal to each other because the Hilbert state of states is separable. In this aspect they resemble the overcomplete set of coherent states of the harmonic oscillator. For cosmic strings an evolving-wormhole wave packet was constructed leading to a dynamically open universe, although the spatial geometry is closed. If the initial state of the Universe is sufficiently localized near the classical singularity, the scale factor is enormously large immediately after the initial instant, a phenomenon described in the literature as "inflation without inflation". In the radiation case, with the help of the exact propagator we constructed wave packets that are examples of dynamic quantum wormholes. The behaviour of the scale factor was studied according to the Bohm-de Broglie interpretation of quantum mechanics. In all three cases $(k=0, \pm 1)$ the singularity is prevented by a quantum force inversely proportional to the cube of the scale factor. Also, in all three cases, the integral $\int_{-\infty}^{t} d \tau^{\prime} / R\left(\tau^{\prime}\right)$ is divergent, so that for the states considered the models have no particle horizon.

\section{Acknowledgment}

The authors thank the Conselho Nacional de Desenvolvimento Científico e Tecnológico (CNPq), Brazil, for finantial support. Special thanks are due to F. G. Alvarenga and J. C. Fabris for discussions and for reading a preliminary version of the manuscript. 


\section{References}

[1] B. S. DeWitt, Phys. Rev. 160, 1113 (1967).

[2] S. W. Hawking and D. B. Page, Phys. Rev. D42, 2655 (1990).

[3] N. A. Lemos and F. G. Alvarenga, Gen. Relat. Grav. 31, 1743 (1999).

[4] F. G. Alvarenga, J. C. Fabris, N. A. Lemos and G. A. Monerat, Gen. Relat. Grav. 34, 651 (2002).

[5] B. F. Schutz, Phys. Rev. D2, 2762 (1970); D4, 3559 (1971).

[6] V. G. Lapchinskii and V. A. Rubakov, Theor. Math. Phys. 33, 1076 (1977).

[7] N. A. Lemos, Class. Quantum Grav. 8, 1303 (1991).

[8] J. Feinberg and Y. Peleg, Phys. Rev. D52, 1988 (1995).

[9] L. J. Garay, Phys. Rev. D44, 1059 (1991).

[10] L. J. Garay, Phys. Rev. D48, 1710 (1993).

[11] G. A. Mena Marugán, Phys. Rev. D50, 3923 (1994).

[12] F. B. Hildebrand, Advanced Calculus for Applications (Prentice-Hall, Englewood Cliffs, NJ, 1976), Section 4.10.

[13] I. S. Gradshteyn and I. M. Ryzhik, Tables of Integrals, Series and Products (Corrected and Enlarged Edition, Academic, New York, 1980).

[14] F. J. Tipler, Phys. Rep. 137, 231 (1986).

[15] N. A. Lemos, J. Math. Phys. 37, 1449 (1996).

[16] P. R. Holland, The Quantum Theory of Motion (Cambridge University Press, Cambridge, 1993). 